\title{
PERFORMANCE OF TRANSMISSION CONTROL PROTOCOL CONGESTIONCONTROL ALGORITHMS ON TELEDESIC SATELLITES CONSTELLATIONS
}

\author{
${ }^{1}$ M. Nirmala and ${ }^{2}$ Ramachandra V. Pujeri \\ ${ }^{1}$ Department of Computer Applications, Hindusthan College of Engineering and Technology, Coimbatore, India \\ ${ }^{2}$ KGiSL Institute of Technology, Coimbatore, India
}

Received 2013-07-07; Revised 2014-01-26; Accepted 2014-04-05

\begin{abstract}
Satellite networking is different from wired or wireless networks. TCP/IP performance and behavior with respect to normal wireless network and wired networks are different from one another. As the architecture of TCP/IP protocol was not designed to perform well over high-latency or noisy channels and so its performance over satellite networks are totally different. The properties of satellite constellations such as the deployment height, motion, direction, link capacity-all differ from one satellite constellations to another. So, certainly the behavior of TCP/IP will considerably differ from one satellite constellations than another and the impact of TCP on the performance of satellite communications was discussed. In this study, we are evaluating the performance of different TCP Congestion algorithms namely Vegas, Reno and Bic on Teledesic satellite constellations. The above mentioned TCP congestion control algorithms are taken for evaluation on the simulated satellite network Teledesic and the performance of the three algorithms under the satellites constellation is measured using suitable metrics to improve the transmission of data packets over satellite links.
\end{abstract}

Keywords: TCP Bic, TCP Reno, Teledesic, TCP Vegas, Performance Metrics

\section{INTRODUCTION}

Satellite Communications delves with a number of technical problems for mobile networks and applications. The difference of properties between satellite link and wired or wireless networks includes larger latency, bursty error characteristics, asymmetric capability and unconventional network architecture. The above metrics creates a greater impact on satellite communication issues (Yongguang, 2003).

Internetworking and computing aspect of satellite networks are major issues in the recent research. A variety of issues involving applications includes, network architecture, medium access controls, multicast routing, asymmetric routing, transport protocols, TCP performance enhancement techniques, data broadcast and information disseminations. Communication over geo-synchronous satellites have long round trip times of approximately $560 \mathrm{~ms}$. The journey through the atmosphere can also introduce bit errors in the data stream. This means that considerable time is needed in Slow Start mechanism in order to open a new connection for TCP, to increase its throughput to what the link capacity will bear. After an errored packet is inferred as lost due to congestion, the congestion window is reduced due to large RTT which results in the slowdown of high rate of throughput. In both cases, expensive satellite link capacity is not fully utilized (Wood et al, 2001).

\section{PROBLEM SPECIFICATION}

The Transmission Control Protocol (TCP) was originally designed for reliable data transport within the terrestrial wired networks. Even though it was not designed for satellite network communication, due to

Corresponding Author: M. Nirmala, Department of Computer Applications, Hindusthan College of Engineering and Technology, Coimbatore, India 
the increasing interest of fixed and mobile interactive service delivery via satellite directly to the final user, the TCP/IP is being studied to overcome its limitations within the satellite medium. TCP congestion control algorithms which were designed originally for wired network communication required only a little modification and performance tuning to be adapted for a Mobile Ad hoc Network. The reasons are the way in which routing and hand-off were. The main aim of this study is to investigate the behavior of some of the existing TCP congestion control algorithms (Vegas, Reno and BIC) under teledesic satellite constellations. The results of this evaluation will make us understand the behavior of TCP congestion control algorithms on satellite network and show some possibilities for improving the existing congestion control algorithm to suit them with satellite networking.

\subsection{The Satellite Constellations (Hu and Vok 2001)}

A group of artificial satellites working together in concert is known as a satellite constellation. They are classified into 3 types based on its height from the earth's surface. They are mentioned below as

- Low Earth Orbit (LEO) satellite constellations

- Medium Earth Orbit (MEO) satellite constellations

- High Earth Orbit (HEO) Satellite constellations

\subsection{Software Used for Satellite Communications Simulation}

\subsubsection{Network Simulator (NS-2) (Grega et al., 2005; Varadhan, 2002)}

NS-2 is a discrete event network simulator. Monarch Group at CMU has extended NS-2 to support wireless networking. NS-2 offers simulation of different kinds of networks, modelling of different types of nodes, protocols, links, traffic and mobilities. NS2 can be used for exact satellite network simulation with a detailed modeling of radio frequency characteristics such as interference, fading, protocol interactions such as interactions of residual burst errors on the link with error checking codes and second-order orbital effects like precession, gravitational anomalies.

\subsection{Satellite Handoff Modeling in NS-2 (Dharma and Zeng, 2003)}

The non-geostationary characteristics and high speed movement of LEO Satellites, has raised to
Satellite handoff modeling, which is a key component of LEO satellite network simulations. Transfer of connection to a new spot beam or satellite is called handover. Various types of handover in satellite systems are mentioned below.

The default network visualization tool of NS-2- the Network Animator (NAM) will not support satellite network visualization.

But there are other open source softwares mentioned below which can be used to visualize satellite constellations:

- $\quad$ Sat-plot-scripts (perl scripts)

- $\mathrm{SaVi}$ (Satellite Constellations Visualization)

- Geomview

\subsection{Sat-Plot-Scripts}

\section{(http://personal.ee.surrey.ac.uk/Personal/ L.Wood/ns/sat-plot-scripts/)}

These Perl scripts can be used to visualize ns satellite constellation configurations, both an overall snapshot of the satellite positions at a given time and the path that a packet takes through the network. Original scripts were written by Tom Henderson, Daedalus Group, University of California at Berkeley. Enhanced (functionality, switches, map options, polar plots) by Wood, (2011) Centre for communication systems research, university of surrey.

\subsection{SaVi (Wood, 2011)}

The Satellite Visualization ( $\mathrm{SaVi}$,) tool, is a computer program for visualizing and animating the movement of satellites and their coverage. This tool has been used for research in academic papers and also proven useful for demonstrating aspects of satellite constellations and their geometry, coverage and movement.

It has a graphical user interface which includes the following features:

- Three-dimensional visualization of satellites in orbit around the Earth

- Display of Satellites footprints on earth's surface and computation of fraction of the earth's surface covered by the constellation

- Simulation of a view of the satellites overhead from an arbitrary point on the earth's surface

\subsection{Geomview (2007)}

Geomview is an open source interactive geometry viewing program built upon the Object Oriented Graphics Library. SaVi leverages Geomview (2007) for simple three-Dimensional (3D) rendering and OpenGL texture mapping. 


\section{TCP CONGESTION ALGORITHMS}

In satellite networks, TCP throughput decreases because the long propagation delays cause longer duration of the Slow Start phase during which the sender may not use the available bandwidth (Akyildiz et al., 2001). Further, it was initially designed to work in networks with low link error rates, i.e., all segment losses were mostly due to network congestion. As a result, the sender decreases its transmission rate each time a segment loss is detected. This causes unnecessary throughput degradation if segment losses occur due to link errors, as it is common in satellite networks.

\subsection{Evaluated TCP Congestion Algorithms (Garg, 2007)}

The congestion control mechanism is realized in 2 ways:

- Reactive Congestion Control: In this, the sender rectifies the congestion window when the network situation becomes marginal or has crossed the threshold

- Proactive Congestion Control: In this type, the feedbacks from the network, guide the sender to reallocate network resources in order to prevent congestion

\subsubsection{TCP Reno Congestion Control (Reno) (Jamal and Sultan, 2008)}

TCP Reno is a Reactive approach, it implements the TCP's Additive Increase Multiplicative Decrease (AIMD) mechanism of increasing the congestion window $\mathrm{W}$ by one segment per Round-Trip Time (RTT) for each received ACK and halving the congestion window $\mathrm{W}$ for each loss event per RTT.

TCP Reno controls the congested window as follows Equation 1 and 2:

$$
\text { Increase }: \mathrm{W}=\mathrm{W}+\frac{1}{\mathrm{~W}}
$$

$$
\text { Decrease }: \mathrm{W}=\mathrm{W}-\frac{1}{2} \mathrm{~W}
$$

\subsubsection{Binary Increase Congestion Control for TCP (BIC)(Rhee and Xu, 2005)}

Binary Increased Congestion control is an implementation of TCP with an optimized congestion control algorithm for high speed networks with high latency. It is a Reactive approach and works on the principle of Loss based congestion control. BIC-TCP sets a minimum window size wmin, it does not have a packet loss for one RTT period and maximum window size wmax, where TCP has packet loss. It uses a Binary search algorithm to reach the target window size wtarget. Which is a midpoint between wmin and wmax Equation 3:

wtarg et $=\frac{(\mathrm{w} \min +\mathrm{w} \max )}{2}$

After the window grows to the mid-point and if no packet loss occurs in the network, then it means that the network can still handle more traffic and thus BICTCP sets the mid-point to be the new minimum (wmin $=\mathrm{w}$ ) and a new target is computed. This has an effect of growing the window really fast when the current window size is far from the available capacity of the path and furthermore, if it is close to the available capacity (where we had the previous loss), it slowly reduces its window increment.

\subsubsection{TCP Vegas Congestion Control (Vegas) (Jamal and Sultan, 2008)}

TCP Vegas is a Proactive approach which is Delay based TCP Congestion control algorithm. It emphasizes packet delay, rather than packet loss as a signal to determine the rate at which to send packets. It adopts a more sophisticated bandwidth estimation scheme. It uses the difference between expected throughput and actual throughput to estimate the available bandwidth in the network. The algorithm heavily depends on accurate calculation of the Base RTT. Base RTT is calculated as the minimum of all measured RTTs, it is commonly the RTT of the first segment sent by the connection.

If the network is not over flown by the traffic, the two parameters and its difference are calculated as follows Equation 4 to 6:

Expected Throughput $=\frac{\text { window } \text { Size }}{\text { Base } \mathrm{RTT}}$

Actual Throughput $=\frac{\text { window Size }}{\text { RTT }}$

Difference $=\left(\begin{array}{c}\text { Expected } \\ \text { Throughput }\end{array}\right)-\left(\begin{array}{c}\text { Actual } \\ \text { Throughput }\end{array}\right) \cdot$ Base RTT 
The idea is that when the network is not congested, the Actual Throughput will be close to the Expected throughput. Otherwise, the actual throughput will be smaller than the expected throughput. TCP Vegas, using this difference in throughputs, estimates the congestion level in the network and updates the window size accordingly. Note that this difference in the throughput can be easily translated into the difference between the window size and the number of acknowledged packets during the round trip time.

\section{SIMULATION AND ANALYSIS OF TELEDESIC SATELLITE CONSTELLATION}

Parameters of Teledesic satellite constellations (Varadhan, 2002) are referred from Table 1.

\subsection{Composition of Satellite Nodes}

Each satellite node has one or more satellite network interface stacks, to which channels are connected to the physical layer object in the stack.

\subsection{Components of Network Stack are}

- Link layer

- Medium Access Control (MAC) layer

- Physical layer

The common satellite network parameters with respect to Teledesic Satellite constellations are given in Table 2.

Physical Layer Type, defined in NS2 as Phy/Sat, passes information up and down the stack. MAC layer performs functionalities such a carrier sense, collision detection, collision avoidance. They are implemented in a single MAC object. The class MAC/Sat a basic MAC for links with only one receiver. The link layer functionalities are queuing and link level retransmission. IT is implemented in NS2 by the class LL/Sat and is the only defined representation. The link uses a drop Tail queue type and the length of queue size is 50 packets. The Time to live value is $32 \mathrm{sec}$. The assumed terminal handoff and satellite handoff interval is $10 \mathrm{~ms}$.

The TCP traffic parameters defined in Table 3 assumes the TCP window size as 30000 and the Maximum segment size is set as 1448 . There are 3 TCP sources TCP1, TCP2, TCp3 and 3 TCP sinks as Sink1, Sink2, Sink3 refer Fig. 1. The 2 ground terminals, one set up at Berkeley and one at Chennai and FTP has been set up over a TCP connection.
Table 1. Parameters of teledesic satellite constellation (Rhee and $\mathrm{Xu}, 2005$ )

\begin{tabular}{ll}
\hline Parameter & Teledesic \\
\hline Altitude & $1375 \mathrm{~km}$ \\
Planes & 12 \\
Satellites per plane & 24 \\
Inclination (deg) & 84.7 \\
Interplane separation (deg) & 15 \\
Seam separation (deg) & 15 \\
Elevation mask (deg) & 40 \\
Intraplane phasing & Yes \\
Interplane phasing & $\mathrm{No}$ \\
ISLs per satellite & 8 \\
ISL bandwidth & $155 \mathrm{Mb} / \mathrm{s}$ \\
Up/downlink bandwidth & $1.5 \mathrm{Mb} / \mathrm{s}$ \\
Cross-seam ISLs & Yes \\
ISL latitude threshold (deg) & 60 \\
Queue length & 50 \\
\hline
\end{tabular}

Table 2. Common satellite network parameters

\begin{tabular}{ll}
\hline Parameter & Teledesic \\
\hline Physical Layer Type & Phy/Sat \\
Link Layer & LL/Sat \\
Mac Type & Mac/Sat \\
Queue Type & DropTail \\
Queue Limit & 50 \\
TTL & 32 \\
Terminal Handoff Interval & $10 \mathrm{~ms}$ \\
Satellite Handoff Interval & $10 \mathrm{~ms}$ \\
\hline
\end{tabular}

Table 3. Traffic parameter0073

\begin{tabular}{ll}
\hline Type & Vegas, Reno and BIC \\
\hline PacketSize / MSS & 1448 \\
Initial Window Size & 30000 \\
TCP Sources & 3 \\
TCP Sink & 3 \\
Ground Terminals & 2 \\
Application & FTP \\
\hline
\end{tabular}

\subsection{Teledesic (Sturza, 1995)0}

There is a significant worldwide demand for broadband communications capacity. Teledesic uses a constellation of 924 Low-Earth Orbit (LEO) satellites operating in Ka-band (30/20 GHz). The Teledesic network will provide "fiber-like" service quality, including low transmission delay, high data rates and low bit error rates, to fixed and mobile users around the world. The Teledesic network will be complementary to terrestrial wireless networks. It will be a broadband overlay for narrowband cellular systems, backbone infrastructure for cell site interconnect and backhaul long distance and international connections. The aggregation of voice channels requires low-delay broadband capability. 


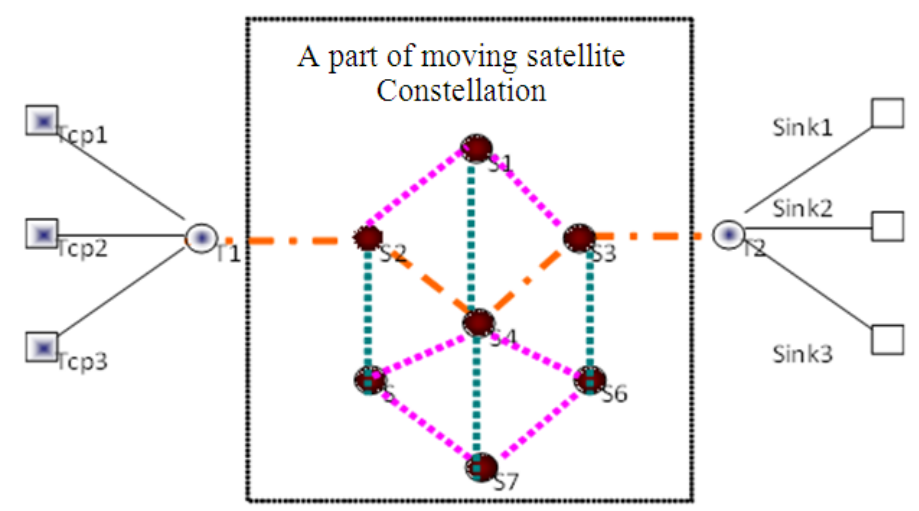

- Ground terminal node

Satellite node

TCP Sources

TPC Sinks

|III Inter place satellite link

IIIIII Intera place satellite link

- Established data path

Fig. 1. The TCP/IP connection Between $T 1$ and $T 2$

Some of the key design drivers of the Teledesic Network are:

- High data rate (broadband) fixed and mobile service

- Continuous global coverage elevation mask angle (deg)

- Fiber-like delay

- Bit error rates less than $10^{-10}$

- Mitigate effects of rain attenuation and blockage

- Rapid network repair

- Geodesic (mesh) network interconnect

\subsection{The TCP Connection Setup}

In order to evaluate the effects of applying different congestion control algorithms in satellite network, the dumbbell network configuration in the following figure is used for the simulation with NS-2. A satellite network which is composed of lot of moving satellites of belonging to a satellite constellation is used to connect two distant ground terminals.
The diagram (Fig. 1) shows an instant of, part of the rapidly changing satellite constellation network. In our simulation, we established communication between two ground terminals. One terminal was setup at Berkeley, California (37.9 lat, -122.3 long) and the another terminal at Chennai, India (13.04 lat, 80.17 long).

The following diagram explains the simulated satellite network connection. In this, the ground terminal $\mathrm{T} 1$ is from which $n$ tcp sources were simulated. T2 is Chennai in which there were tcp sinks. The tcp sources and sinks were connected (terminals T1 and T2) were connected over the highly moving satellite nodes.

Under NS2, there is no visualization support (namnetwork) animation for visualizing a satellite constellation. For that we used a tool called as "SatPlot Scripts". During simulation, one particular instance of our interest can be dumbed into a log file. The dump file will contain information about the satellite location and data path at that instance.

Satplot scripts will use those information and Project satellite locations and data paths on a standard world map. 


\subsection{Performance Metrics}

\subsubsection{Contention Window (cwin)}

To avoid collisions within a traffic category, the station counts down an additional random number of time slots, known as a contention window, before attempting to transmit data. If another station transmits before the countdown has ended, the station waits for the next idle period, after which it continues the countdown where it left off.

The contention window dynamics are more important in a TCP congestion control algorithm. The better contention window management provides better throughput and minimum end to end delay. So, 'time Vs cwin size' is used as an evaluation metric.

\subsubsection{Round Trip Time (RTT)}

In telecommunications, the Round-Trip Delay time (RTD) or Round-Trip Time (RTT) is the length of time it takes for a signal to be sent plus the length of time it takes for an acknowledgment of that signal to be received. This time delay therefore consists of the transmission times between the two points of a signal.

\subsubsection{The Dynamics of Round of Links}

It is the cumulative delay which includes transmission delay, propagation delay and processing delay across a network

\subsubsection{Jitter or Packet Delay Variation (PDV)}

Packet Delay Variation (PDV) is the difference in end-to-end one-way delay between selected packets in a flow with any lost packets being ignored.

The delay is specified from the start of the packet being transmitted at the source to the end of the packet being received at the destination. A component of the delay which does not vary from packet to packet can be ignored, hence if the packet sizes are the same and packets always take the same time to be processed at the destination then the packet arrival time at the destination could be used instead of the time the end of the packet is received.

\subsubsection{Packet Delivery Fraction (PDF)}

PDF is the ratio of received packets by the destination nodes to the total amount of data packets sent by the CBR sources. This metric tells us how reliable the protocol is. It gives the rate of loss which will be undergone by the transport protocol, which will then affect the maximum throughput the ad hoc network will be capable of supporting Equation 7:

$$
P=\frac{1}{c} \sum_{f=1}^{e} \frac{R_{f}}{N_{f}}
$$

Where:

$\mathrm{P}=$ the fraction of successfully delivered packets

$\mathrm{c}=$ the total number of flow or connection

$\mathrm{f}=$ the unique flow id serving as index

$\mathrm{R}=$ the count of packets received from flow $\mathrm{f}$

$\mathrm{Nf}=$ the count of packets transmitted to $\mathrm{f}$

Packet Delivery fraction is also referred as Packet Delivery Ratio (PDR).

\subsubsection{Throughput}

The throughput metric measures, how well the network can constantly provide data to the sink. The amount of packets successfully received at the destination. This is a fundamental measure of the performance of a network and therefore, an important factor to consider.

\subsubsection{Dropped Packets}

The number of data packets that are not successfully sent to the destination during the transmission. Here we calculate time Vs number of packets dropped contention window (cwin)

\section{RESULTS}

The various metrics used for evaluation of transport protocol includes Contention window, RTT, End to end delay, Jitter, PDF, throughput and dropped packets. Based on the metrics taken the simulated results using NS2 has been plotted as graphs.

\section{DISCUSSION}

The previous research made on Iridium satellite constellations (Nirmala and Pujeri, 2012)0 has proved the results of Vegas to be good and the comparative analysis among two satellite constellations Teledesic and Iridium will explore better data transmission over satellite networks.

The performance of the congestion control algorithms on Teledesic satellite constellations has been evaluated. The following line graphs and bar charts shows the comparative performance of the three algorithms namely TCP vegas, BIC and Reno under Teledesic satellite constellation. 


\subsection{The Analysis of Contention Window and RTT Dynamics}

Generally, under wired networks, we may see rhythmic patterns in the estimation of contention window size as well as the round trip time. Under MANET, we will not get any rhythmic changes in RTT and contention window size due to the random mobility and random link failures. But, if we see the contention window dynamics and the RTT dynamics of tcp congestion control algorithms under satellite constellation, it is entirely different from that of a mobile ad hoc network scenario and resemble that of a wired network scenario. It is a most interesting observation-in fact, we didn't expect such a rhythmic pattern under the highly changing satellite constellation. The reason for this rhythmic behavior is the hand-off and routing strategies used in the present satellite networks-they were not like as in the case of normal ad hoc network.

The line graph (Fig. 2) shows the contention window dynamics of the three congestion control algorithms under Teledesic satellite constellation. As shown in the chart, there is no change in contention window size in the case of Vegas.

The round trip time in the case of Vegas was very minimum is shown in (Fig. 3)

\subsection{The Analysis of End to End Delay and Jitter}

As shown in the (Fig. 4), the performance in terms of end to end delay was very minimum in the case of Vegas.
The bar chart (Fig. 5), shows the average performance in terms of end to end delay. In terms of end to end delay Vegas outperformed the other two algorithms.

The line graph (Fig. 6) shows the performance in terms of jitter (jitter1-Packet delay variation-i.e., the difference in end-to-end delay). The performance in terms of jitter was very minimum in the case of bic.

The bar chart (Fig. 7) shows the average jitter (jitter1). The performance in terms of jitter was good in the case of bic.

\subsection{Analysis of PDF,Throughput and Dropped Packets}

The bar chart (Fig. 8) shows the performance in terms of PDF. With respect ot PDF, the performance of vegas was better than the other two.

The bar chart (Fig. 9) shows another important observation in this evaluation. Bic provided highest throughput under Teledesic constellation.

The bar chart (Fig. 10) shows the time vs packet dropped graph. According to the results, the dropped packet count in the case of vegas was zero. (the following graph was drawn from -; that is why we see a tiny bar for vegas which were actually representing).

According the above results, Bic provided better throughput and minimum jitter. Vegas provided minimum end to end delay.

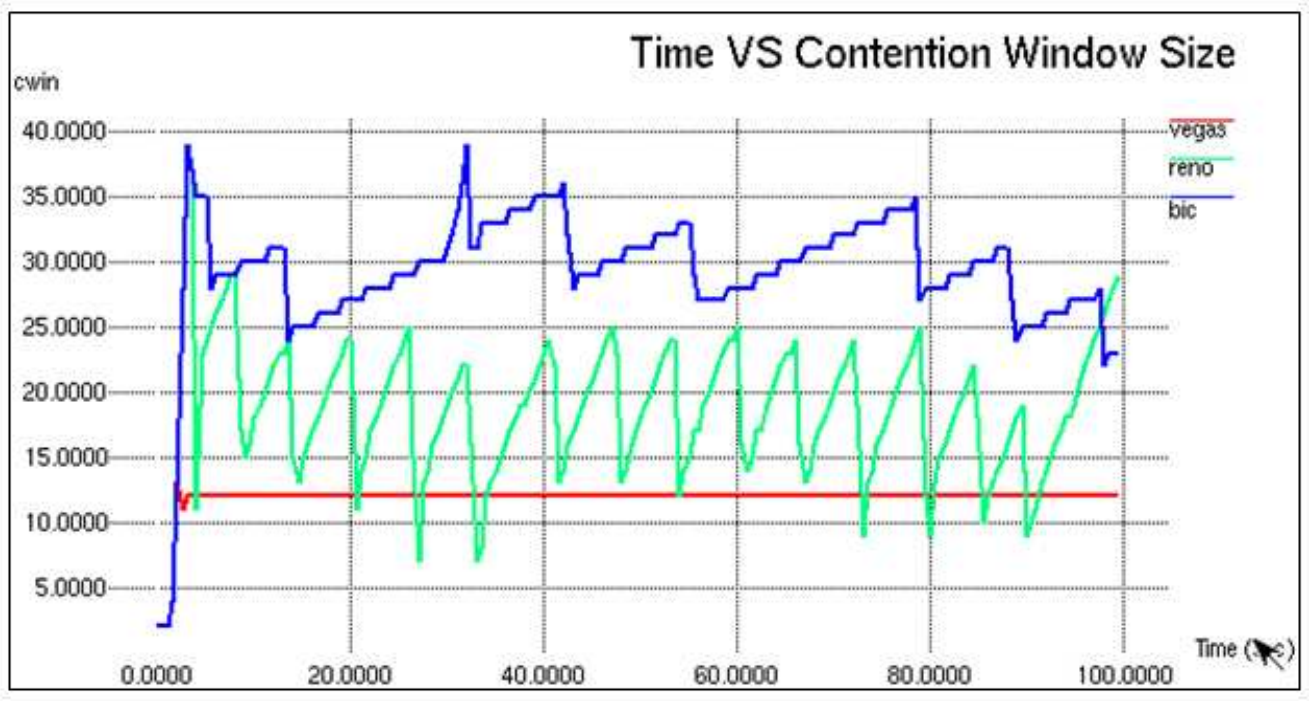

Fig. 2. The contention window dynamics 


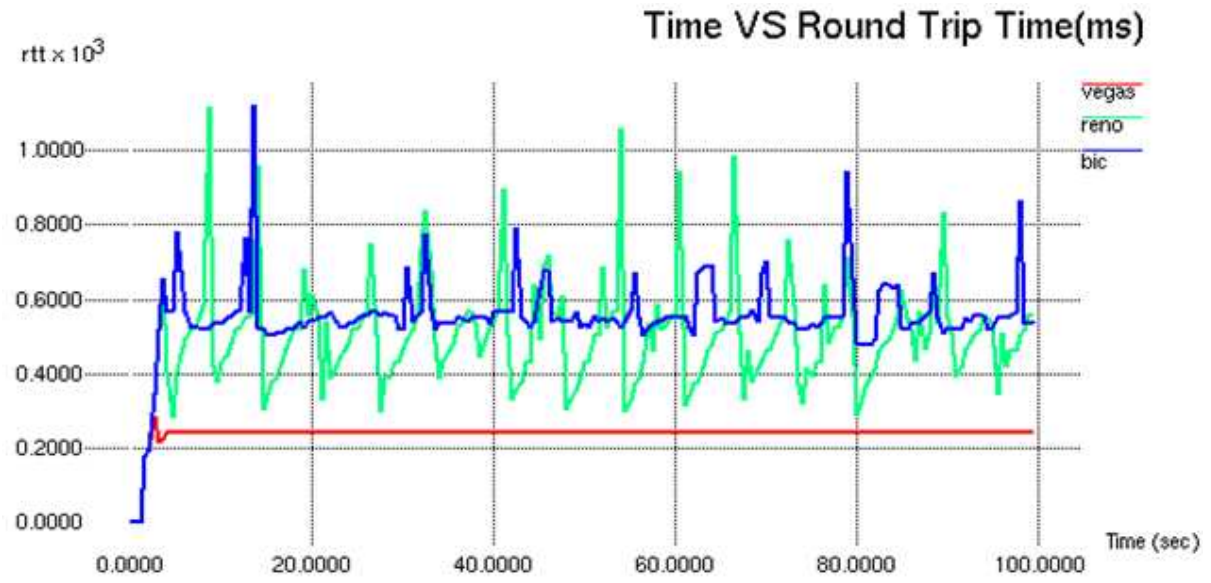

Fig. 3. The RTT dynamics

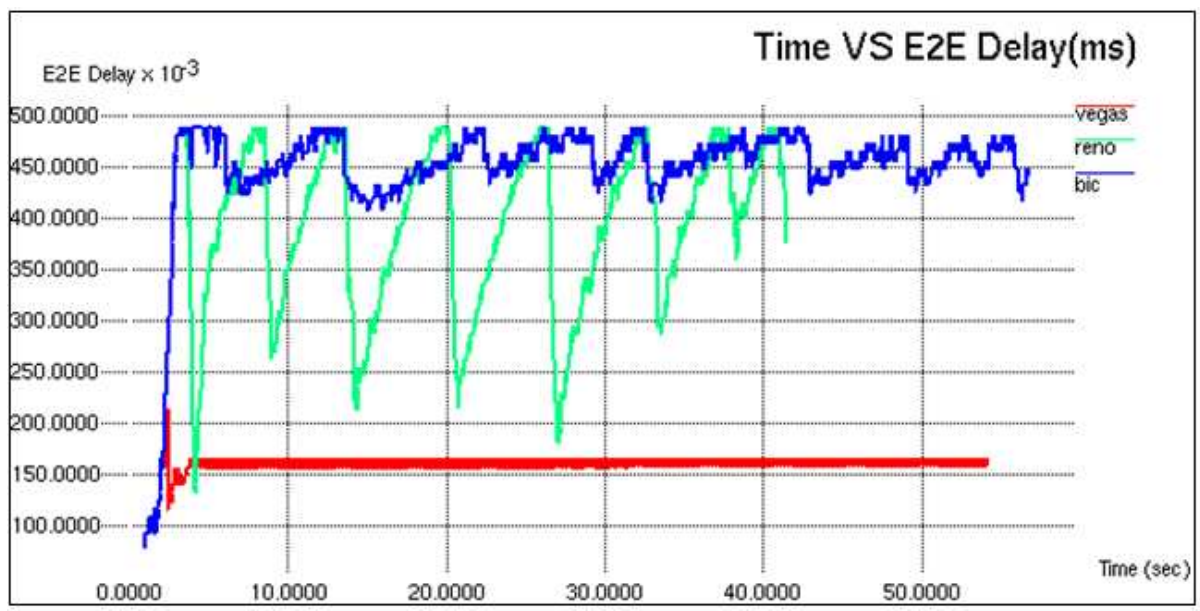

Fig. 4. The time VS E2E graph

E2E Delay (ms)

The Average End to End Delay

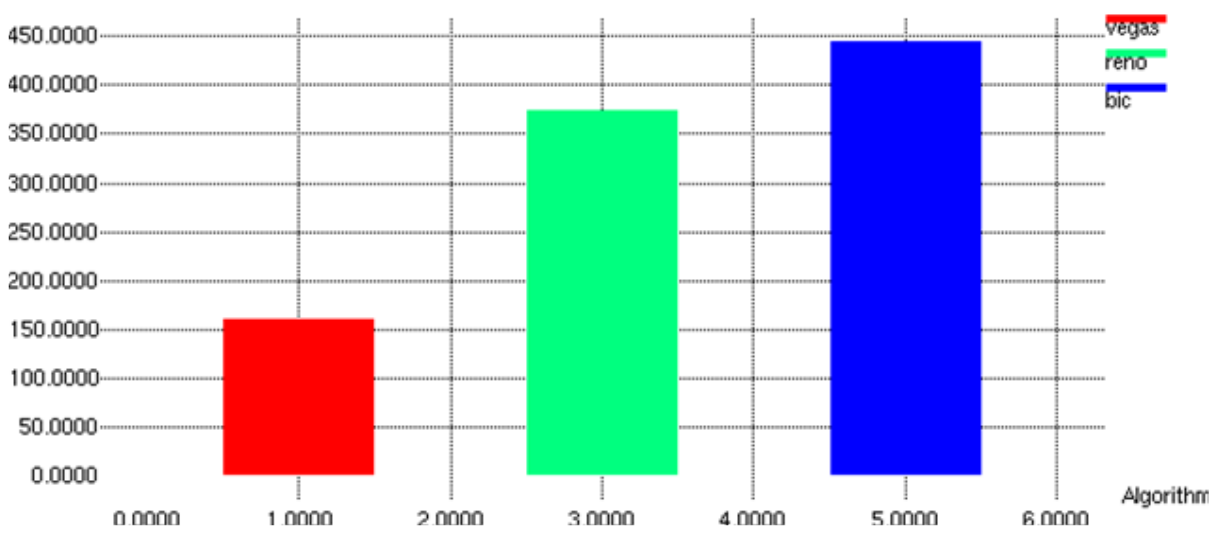

Fig. 5. The average E2E 


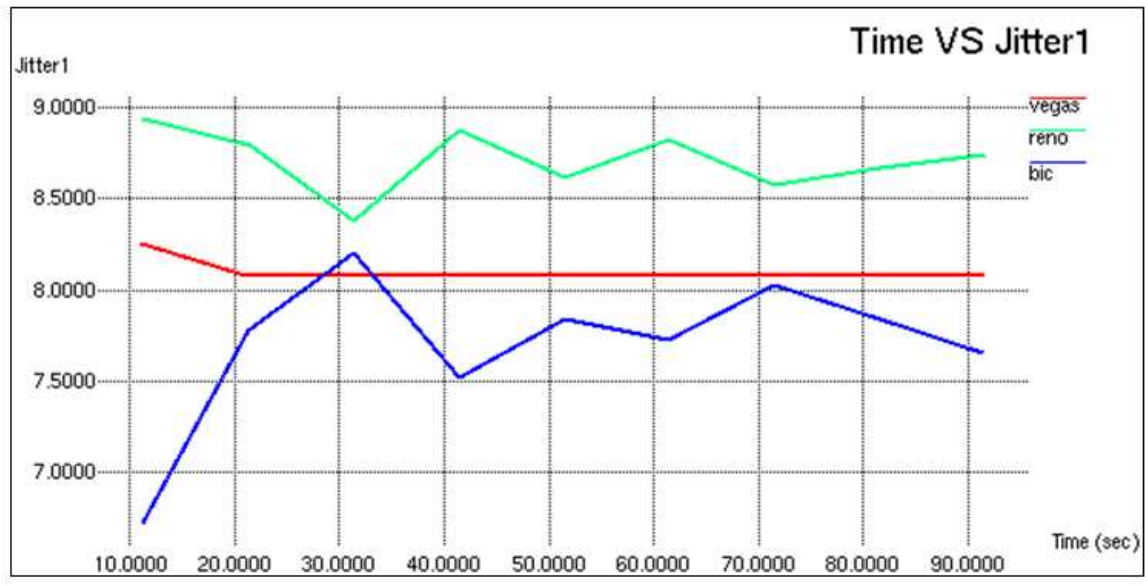

Fig. 6. The time Vs jitter1 (PDV) graph

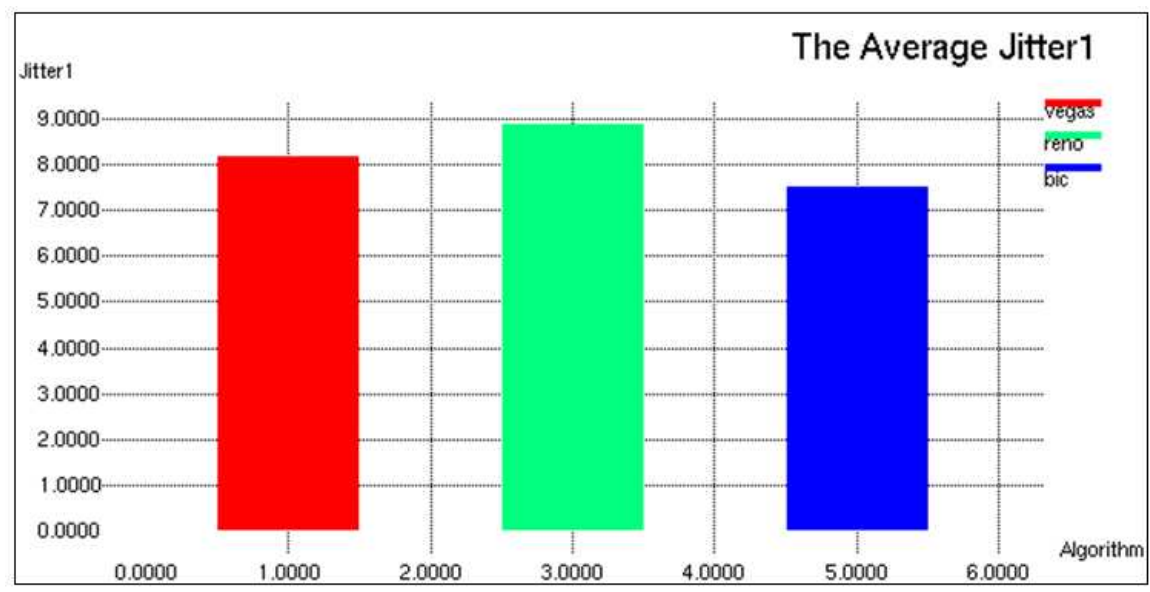

Fig. 7. The average jitter (PDV)

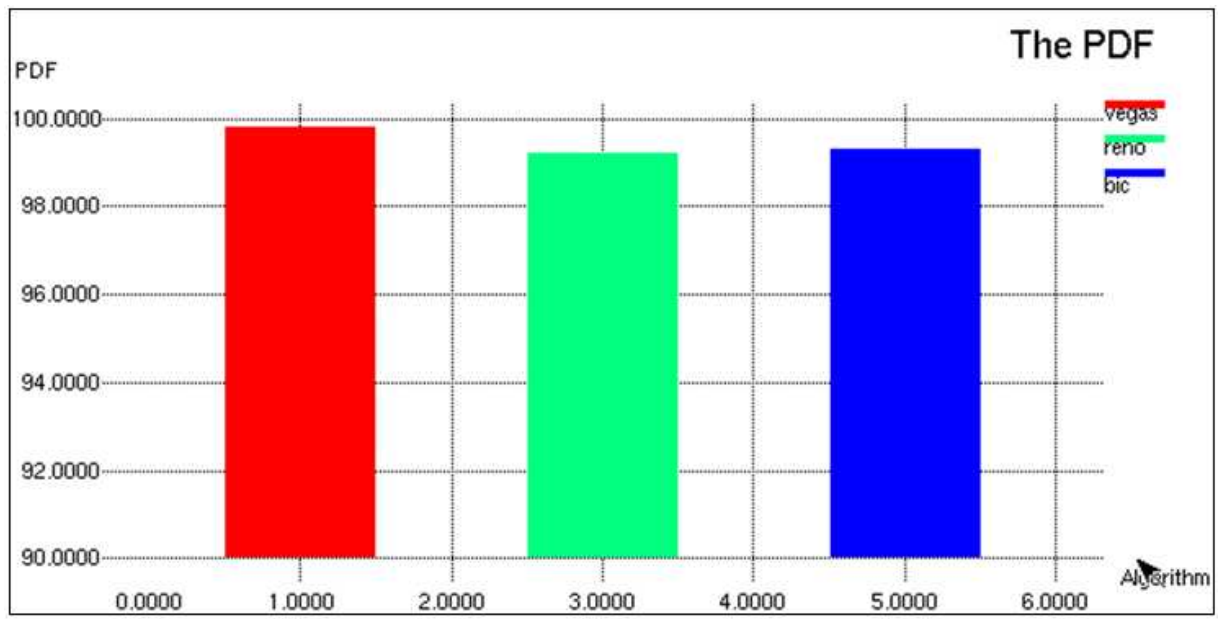

Fig. 8. The average PDF 


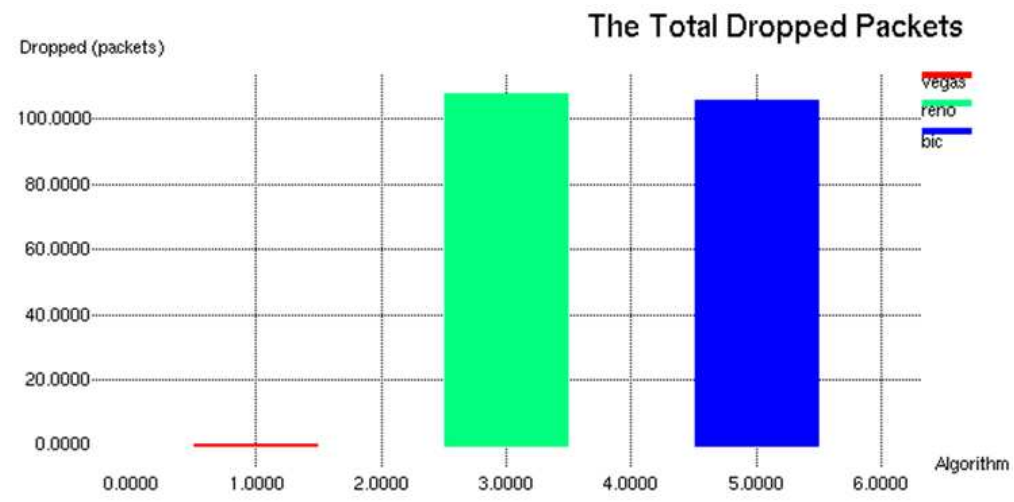

Fig. 9. The total dropped packets

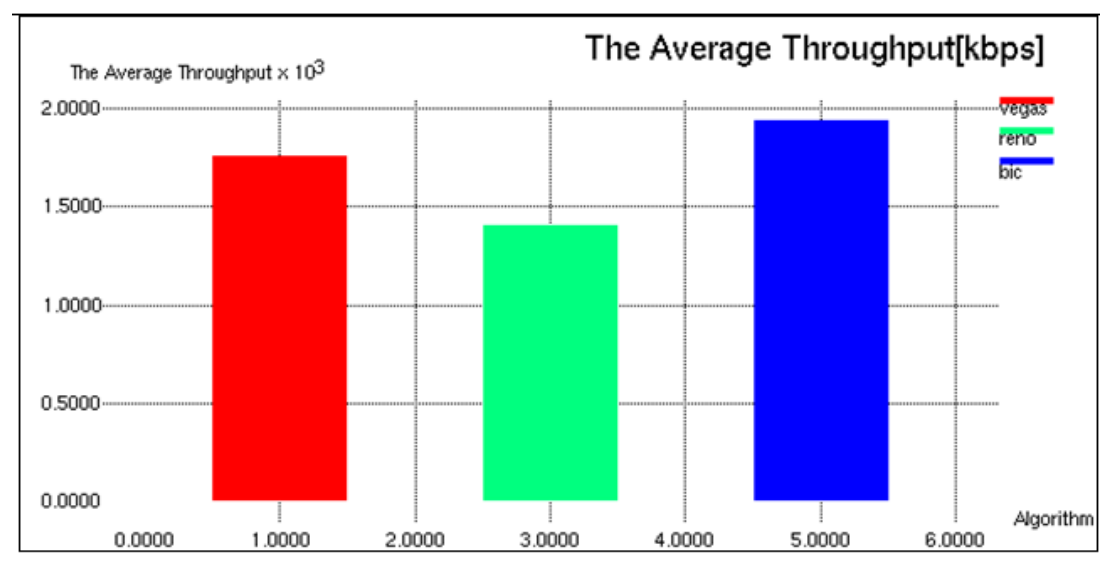

Fig. 10. The average throughput

\section{CONCLUSION}

According to the arrived results, with respect to throughput and jitter the performance of bic was good under Teledesic constellation. but, with respect to some metrics, the performance of vegas was little bit better under bic. The reasons for the better behavior of bic under Teledesic should be explored for designing a better congestion control algorithm exclusively for Satellite Networks.

Irrespective of the high end to end delay, the behavior of TCP/IP under Satellite network is somewhat resembling a high latency wired network. If we see the cwin and rtt dynamics, we can realize the similarity with wired network.

Even though, the communication is happening wirelessly under rapid satellite movements, the behavior of tcp under satellite network is not like a mobile ad hoc network. The reason for this behavior may be the handoff and routing strategies used in the present satellite network systems-they were not like as in the case of normal ad hoc network or wired network. So future works may address the ways to improve the behavior of a TCP congestion control algorithm by considering the hand-off and routing strategies of the satellite networking. TCP protocols have lower throughput, pdf and high end to end delay under satellite networks mainly due to the effects of long propagation delays and high link error rates Future works will address the ways to improve any of the selected tcp congestion algorithm for satellite networking.

\section{ANNEXURES}

The simulated Teledesic Constellation and Terminals-Plotted using "Sat-plot-scripts" has been represented through Fig. 11.

The Coverage of Map Iridium Constellation-An instant Created using the tool "SaVi" has been represented through Fig. 12. In the Fig. 12 the green dots represents the locations of the satellites at that instant. 
M. Nirmala and Ramachandra V. Pujeri / Journal of Computer Science 10 (8): 1531-1542, 2014

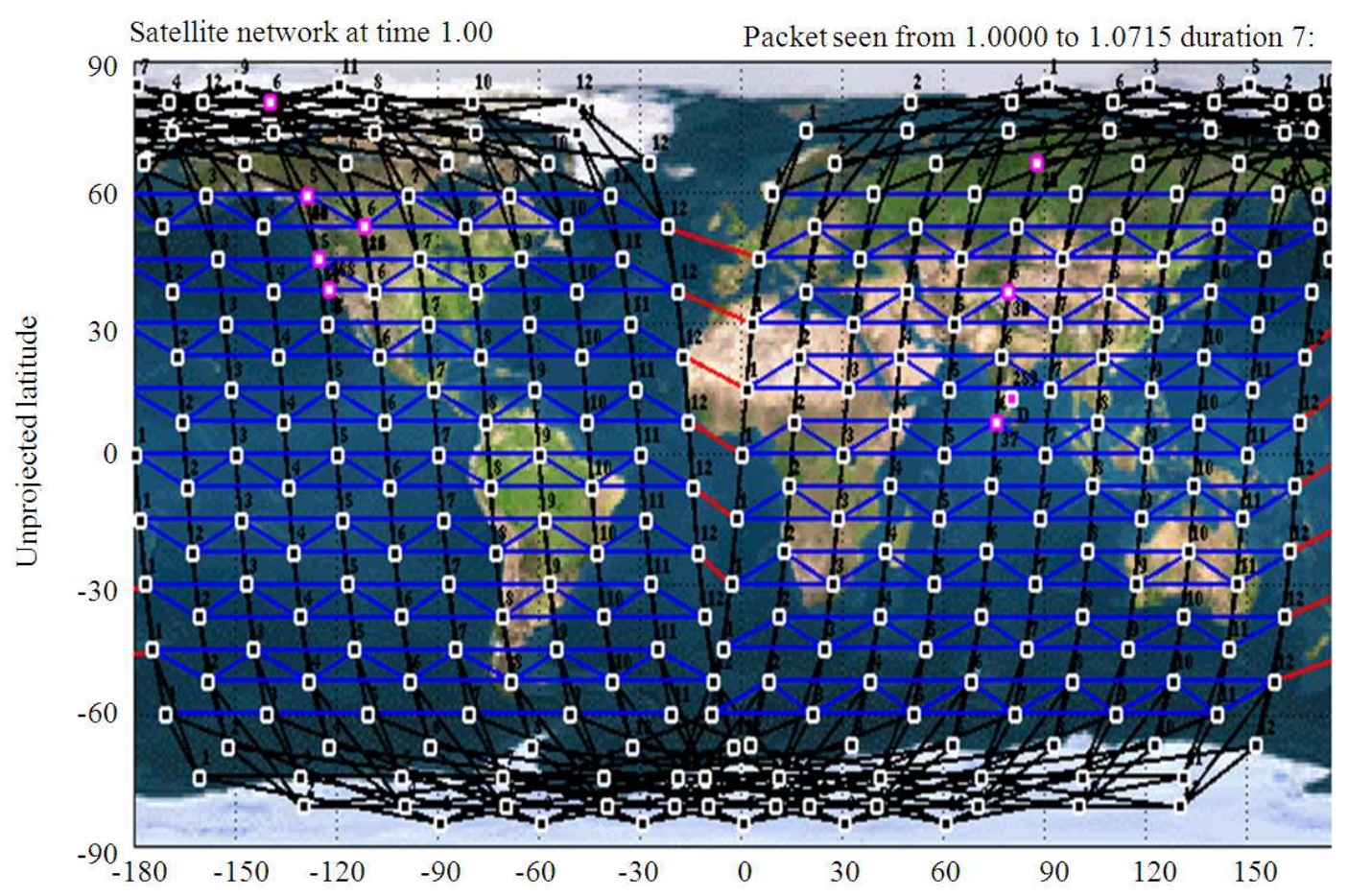

Fig. 11. The teledesic satellites, links and the communication hops (pink) between terminals

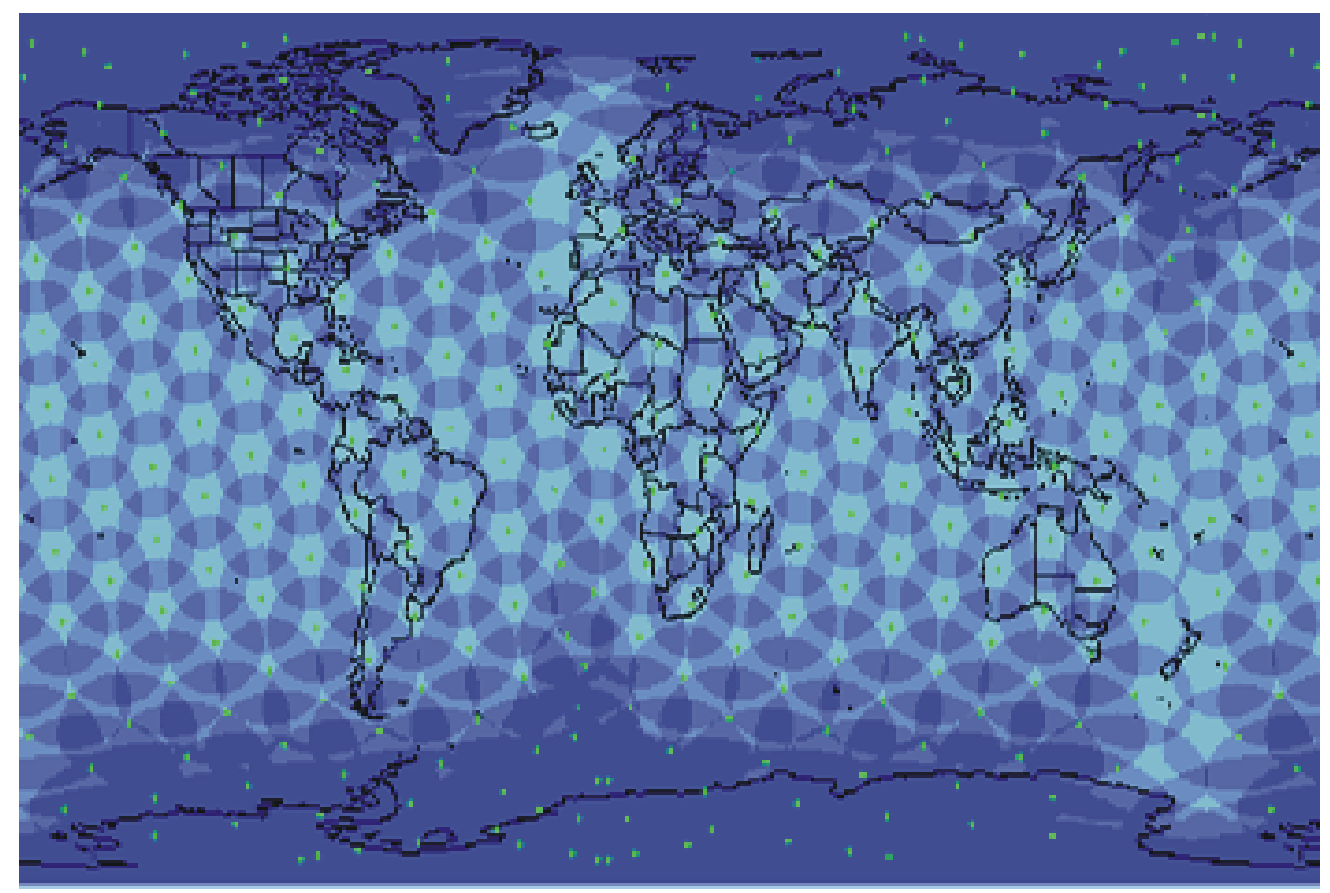

Fig. 12. The teledesic coverage map 


\section{AUTHORS AND AFFILIATIONS}

M. Nirmala, received the BSc Degree in Computer Science from Bharathiar University and MCA from Bharathidasan University. She has a total teaching experience of 12 years. Her area of interest includes Networking, Simulation and Modeling. She is a member of the professional society ACM, CSTA, IAENG.

Ramachandra V. Pujeri, received the $\mathrm{BE}$ in Electronics and Communication Engineering from Karnataka University, Dharwad, ME in Computer Science and Engg from PSG College of Technology, Coimbatore, $\mathrm{PhD}$ in Information and Communication Engineering from Anna University, Chennai, MBA in Human Resource Management, from Pondicherry University, Pondicherry, in 1996, 2002, 2007 and 2008 respectively. He is active life member of ISTE, SSI, MIE, ACS and IEE. His has written three textbooks. He is having around 17 years of teaching experience in the various top ten engineering colleges in India. He is an active expert committee member of AICTE, NBA, DoEACC, NACC and various Universities in India. Currently, under him twenty research scholars pursuing their Ph.D. His research interests lie in the areas of Computer Networking, Operating Systems, Software Engineering, Modeling and Simulation, Quality of Services and Data Mining. Currently, he is working as Vice-Principal of KGiSl Institute of Technology.

\section{ACKNOWLEDGEMENT}

My thanks to Marian GRega Lubomir Copjan, Stanislav Marchevsky, Stanislav Benco and their research paper Possibility of Using Network Simulator (NS-2) for modeling Satellite Networks, Acta Electrotechnica et Informatica No. 4, Vol. 5, 2005 for my thanks to my Research Guide Dr. Ramachandra.V. Pujeri for encouraging me in my research endeavors which covers both technological and social aspects.

\section{REFERENCES}

Akyildiz, I.F., G. Morabito and S. Palazzo, 2001. TCPpeach: A new congestion control scheme for satellite IP networks. IEEE/ACM Trans. Network., 9: 307321. DOI: $10.1109 / 90.929853$
Dharma, P.A. and Q.A Zeng, 2003. Introduction to Wireless and Mobile Systems. 1st Edn., Thomson Brooks/Cole, ISBN-10: 0534408516, pp: 438.

Garg, V., 2007. Wireless Communications and Networking. 1st Edn., Morgan Kaufmann, ISBN10: 0123735807, pp: 840.

Geomview, 2007. Sourceforge project site. Geomview.

GRega, M., L. Copjan, S. Marchevsky and S. Benco, 2005. Possibility of using network simulator (Ns-2) for modeling satellite networks. Acta Electrotechnica et Informatica.

Hu, Y. and L. Vok, 2001. Satellite-based internet: A tutorial. IEEE Commun. Magazine, 39: 154-162. DOI: $10.1109 / 35.910603$

Jamal, H. and K. Sultan, 2008. Performance analysis of TCP congestion control algorithms. Int. J. Comput. Commun., 2: 30-38.

Nirmala, M. and R.V. Pujeri, 2012. Performance of TCP vegas, bic and reno congestion control algorithms on iridium satellite constellations, Int. J. Comput. Netw. Inform. Security, 12: 40-49. DOI: 10.5815/ijcnis.2012.12.04

Rhee, S.H.I and L. Xu, 2005. CUBIC: A new TCPfriendly high-speed TCP variant. Department of Computer Science. DOI: 10.1.1.153.3152\&rep=rep1\&type=pdf

Sturza, M.A., 1995. Architecture of the Teledesic satellite system. Teledesic Corporation.

Varadhan, F.K, 2002. Satellite networking in ns the ns manual.

Wood, L., G. Pavlou and B. Evans, 2001. Satellitebased internet technology and services-effects on tcp of routing strategies in satellite constellations. IEEE Commun. Magazine,

Wood, L., 2011. Examples of use of SaVi, list of papers and articles. Centre for Communication Systems.

Yongguang, Z., 2003. Internetworking and Computing over Satellite Networks. 1st Edn., Springer, ISBN10: 1402074247, pp: 262. 